\section{Extracellular serine feeds cancer skin stem cells}

Stem cells can acquire oncogenic mutations and, in some cases, this can lead to the initiation of neoplasms. The mechanisms underlying the selection of a subset of stem cells to become malignant are largely unknown. Using epidermal stem cells (EpdSCs) as a model to address this question, Baksh et al. found that metabolic reprogramming - which renders EpdSCs dependent on extracellular serine - inhibits their differentiation, thus promoting oncogenic cell proliferation.

EpdSCs reside in the basal (innermost) layer of mammalian epidermis, and balancing their self-renewal and cell differentiation is crucial for the maintenance of the skin barrier. These cells are at the origin of squamous cell carcinomas, which are among the most common types of cancer.

To explore the metabolism of premalignant EpdSCs, the authors generated mice in which epidermal progenitors express SOX2, a transcription factor that is known to promote tumour initiation and progression. As SOX2-expressing stem cells induce a stress response that involves global protein synthesis repression, the authors investigated

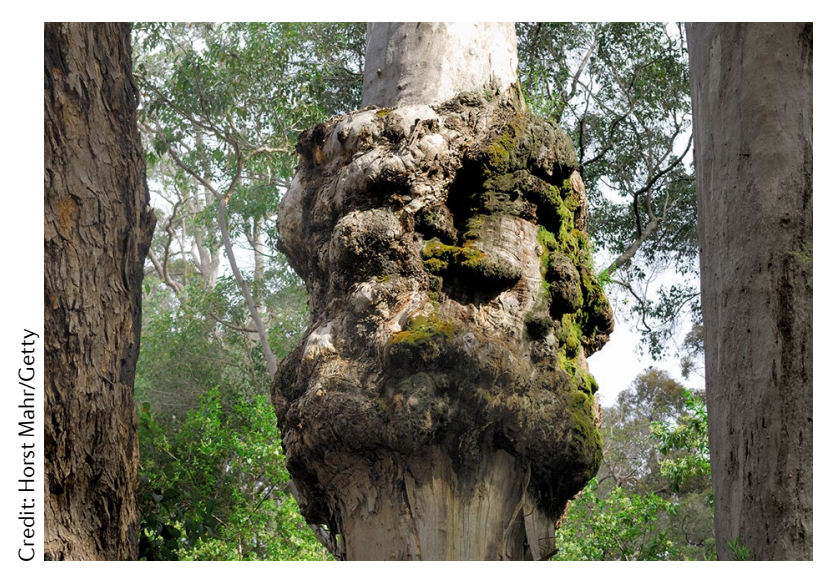

whether premalignant SOX2 ${ }^{+}$EpdSCs have altered amino acid requirements. They found that SOX $2^{+}$EpdSCs consumed serine in greater amounts compared with wild-type EpdSCs (which was unexpected given the reduced translation) and that they relied predominantly on extracellular sources of serine for proliferation. When serine was removed from the culture medium, SOX2 ${ }^{+}$EpdSCs were incapable of maintaining an intracellular pool of serine and stopped proliferating, whereas wild-type cells were unaffected. Intracellular serine synthesis can either occur de novo, through a glucose-dependent pathway, or from the conversion of glycine to serine. Following serine and glycine deprivation, wild-type cells derived the majority of intracellular serine from glucose, whereas premalignant cells did not, indicating that premalignant EpdSCs inhibit glucose-dependent serine synthesis and thus become dependent on extracellular sources of serine to sustain cell proliferation.

When analysing these metabolic differences, the authors found that SOX $2^{+}$EpdSCs are not generally defective in glucose metabolism, but have altered expression of metabolic enzymes that promotes the mitochondrial consumption of pyruvate. In the cytosol, pyruvate is used to produce $\mathrm{NAD}^{+}$, so its diversion to mitochondria leads to depletion of cytosolic $\mathrm{NAD}^{+}$. As $\mathrm{NAD}^{+}$is also a cofactor for the rate-limiting enzyme required for glucose-dependent serine synthesis, $\mathrm{NAD}^{+}$depletion supplementing pyruvate to increase cytosolic $\mathrm{NAD}^{+}$levels eliminated the dependency of premalignant EpdSCs lowering $\mathrm{NAD}^{+}$in wild-type cells impairs serine synthesis. Accordingly, on extracellular serine. Conversely, made them dependent on extracellular serine. Thus, cytosolic NAD ${ }^{+}$ levels regulate serine biosynthesis in EpdSCs.

To assess how extracellular serine affects EpdSC function in vivo, $\mathrm{SOX}^{+}$and wild-type mice were placed on a serine/glycine-free diet. The proliferation of SOX2 ${ }^{+}$EpdSCs was impaired in a cell-autonomous manner. However, the differentiating layers of wild-type epidermis became thickened on a serine/glycine-free diet, as a consequence of premature EpdSC differentiation, whereas SOX2 ${ }^{+}$EpdSCs showed no signs of increased differentiation. These observations indicate that serine availability controls the balance between proliferation and differentiation and that while serine deprivation forces differentiation in wild-type cells, premalignant cells are resistant to differentiation. Mechanistically, de novo serine biosynthesis was found to induce the accumulation of $\alpha$-ketoglutarate ( $\alpha \mathrm{KG}$ ), which activates $\alpha \mathrm{KG}$-dependent dioxygenases, including histone demethylases. Accordingly, serine biosynthesis led to a reduction in histone 3 lysine 27 trimethylation - known to repress terminal cell differentiation in the skin - and increased cell differentiation.

Forcing stem cell differentiation is an effective tumour-suppressive mechanism, and indeed serine/glycine starvation impaired tumour initiation in mice. But crucially, such a repressive mechanism required a functional de novo serine synthesis pathway, which becomes silenced in premalignant EpdSCs. The finding that wild-type and premalignant EpdSCs differ in their dependence on environmental serine raises the possibility of limiting serine uptake as a therapeutic strategy to inhibit or eliminate oncogenic stem cells.

Kim Baumann

ORIGINAL ARTICLE Baksh, S. C. et al. Extracellular serine controls epidermal stem cell fate and tumour initiation. Nat. Cell Biol. https://doi.org/10.1038/ s41556-020-0525-9 (2020) 